SHORT REPORT

\title{
Altered subthalamo-pallidal synchronisation in parkinsonian dyskinesias
}

\author{
G Foffani, G Ardolino, B Meda, M Egidi, P Rampini, E Caputo, G Baselli, A Priori
}

J Neurol Neurosurg Psychiatry 2005;76:426-428. doi: 10.1136/jnnp.2004.043547

The aim of this work was to study the role of subthalamopallidal synchronisation in the pathophysiology of dyskinesias. We recorded local field potentials (LFPs) in a patient with Parkinson's disease and left surgery induced dyskinesias with double, bilateral deep brain stimulation electrode implants in the subthalamic nucleus (STN) and the globus pallidus internus (GPi). Synchronisation was studied through coherence analysis. In the nuclei contralateral to the dyskinetic side of the body there was decreased STN-GPi coherence in the high beta range $(20-30 \mathrm{~Hz})$ and an enhanced coherence at low frequencies $(<10 \mathrm{~Hz})$. Despite the possible limitations arising from single-case observations, our findings suggest that parkinsonian dyskinesias are related to altered synchronisation between different structures of the basal ganglia. Firing abnormalities within individual basal ganglia nuclei are probably not enough to account for the complex balance between hypokinetic and hyperkinetic symptoms in human parkinsonian dyskinesias and altered interactions between nuclei should also be considered.

$\mathrm{D}$ yskinesias frequently complicate Parkinson's disease and appear in most patients treated with levodopa within 5 years. Transient contralateral dyskinesias also sometimes appear after the implantation of deep brain stimulation (DBS) electrodes in the subthalamic nucleus (STN) possibly due to a transient lesion effect of the surgical procedure. Despite the clinical relevance of dyskinesias in the management of Parkinsons's disease, their pathophysiology remains unclear. ${ }^{1-6}$ Single-neuron studies described abnormal firing patterns in the globus pallidus internus (GPi) and the STN of parkinsonian monkeys and human patients with dyskinesias. ${ }^{7-10}$ Local field potential (LFP) recordings from DBS electrodes implanted in the GPi and STN of parkinsonian patients revealed complex patterns of oscillatory STN-GPi activity, from the classical EEG frequency range ${ }^{11-15}$ up to surprisingly high frequencies. ${ }^{16}$ Oscillatory LFP activity has also been described in the GPi of dystonic patients. ${ }^{17}$ However, no information is available about LFPs specifically related to dyskinesias in Parkinson's disease. We hypothesised that the complex balance between hypokinetic and hyperkinetic symptoms in parkinsonian dyskinesias could arise not only from the abnormal activity within individual structures of the basal ganglia but also from an altered synchronisation between them. We tested this hypothesis by recording LFPs bilaterally from both the STN and the GPi in a parkinsonian patient with post-surgery dyskinesias mainly involving the left upper limb, and studying STN-GPi synchronisation.

\section{METHODS}

The patient

The patient was a 54 year old woman with 14 year history of rigid-akinetic Parkinson's disease. The onset of the disease was on the left side of the body. She was on levodopa+dopamine agonists therapy for a daily dose of $1500 \mathrm{mg}$ levodopa equivalents. She had a previous bilateral GPi DBS implant that was poorly effective and she therefore received a bilateral STN DBS implant after fulfilling specific inclusion criteria. ${ }^{18}$ Soon after implantation of the DBS electrodes in the STN she developed dyskinesias mainly at the left upper limb, which were worsened by levodopa. The phenomenology of dyskinesias resembled involuntary movements triggered by levodopa before surgery. On the right side there was no dyskinesia. Despite these transient dyskinesias, which lasted less than a week, the DBS had a good long term outcome. Before the STN surgery, the patient motor performance measured by Unified Parkinson's Disease Rating Scale (UPDRS) part III was 62 "off" therapy and 23.5 "on" therapy. At 1 year after surgery the UPDRS III "off" medication "on" STN stimulation was 19. Dyskinesias and fluctuations (UPDRS IV part A and B, respectively) were reduced from 7 and 7 before the surgery to 1 and 2 , respectively, at 1 year after surgery. The patient was studied after informed consent and local ethical committee approval.

\section{Electrode localisation}

The pre-operative CT-MRI targeting of the STN, intraoperative clinical and neurophysiological procedures, postoperative CT-MRI electrode confirmation, and clinical evaluation of efficacy are described in detail elsewhere. ${ }^{16}{ }^{19-21}$ As in our previous work, ${ }^{15-16}$ the STN electrodes were verified to be within (or close to) the nuclei. Using the same imaging techniques, the distal contacts of the pallidal electrodes were confirmed to be within the GPi. Postoperative neuroimaging ruled out significant asymmetries of electrode positions and/or of lesion effect induced by the surgical procedures.

\section{Postoperative recordings}

The patient was comfortably seated in an armchair. LFPs were recorded from the STN and GPi ipsilateral electrodes (3389 Medtronic, contacts $0-1$ and 0-2, respectively; Medtronic, Minneapolis, MN, USA) after overnight withdrawal of dopaminergic medication. The patient was asked to maintain a totally relaxed position, but she had spontaneous dyskinetic jerks on the left side of the body almost continuously during the recording session. As a control, LFPs were also recorded on the left side of the brain at rest and during voluntary contractions of right forearm muscles. Signals were preamplified, differentially amplified, and filtered (2-1000 Hz) through a CED 1902 amplifier (Cambridge Electronic Design, Cambridge, UK), A/D converted (sampling rate $2500 \mathrm{~Hz}$ ) through a CED 1401 interface (Cambridge Electronic Design), on-line analysed on a personal computer, and stored using CED Signal software

Abbreviations: DBS, deep brain stimulation; GPi, globus pallidus internus; LFPs, local field potentials; STN, subthalamic nucleus; UPDRS, Unified Parkinson's Disease Rating Scale 

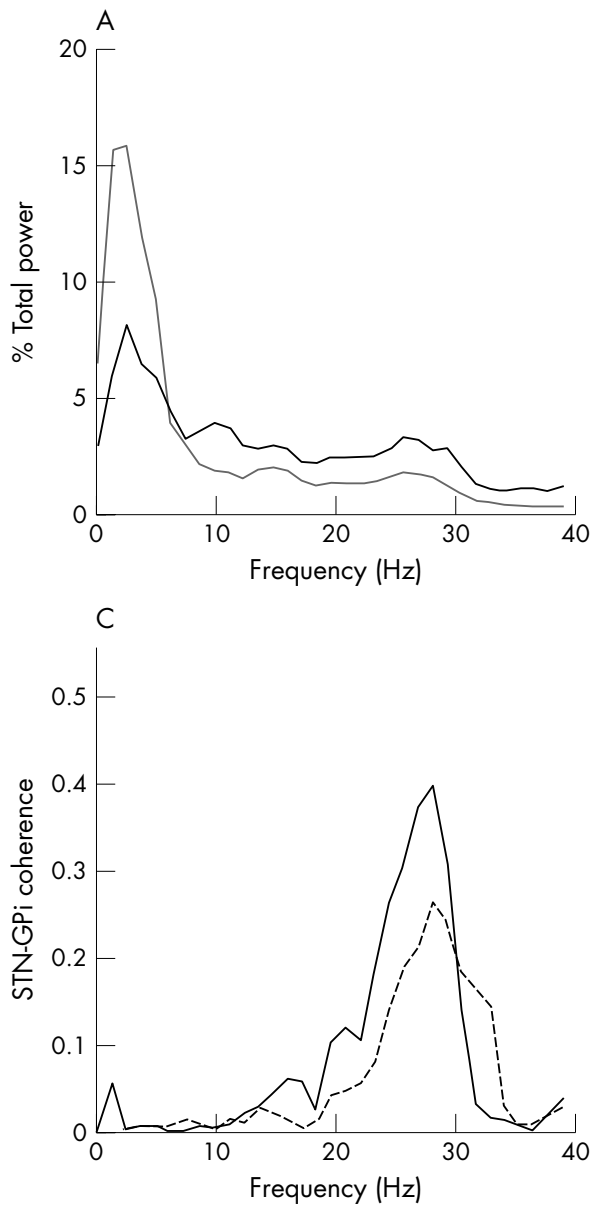
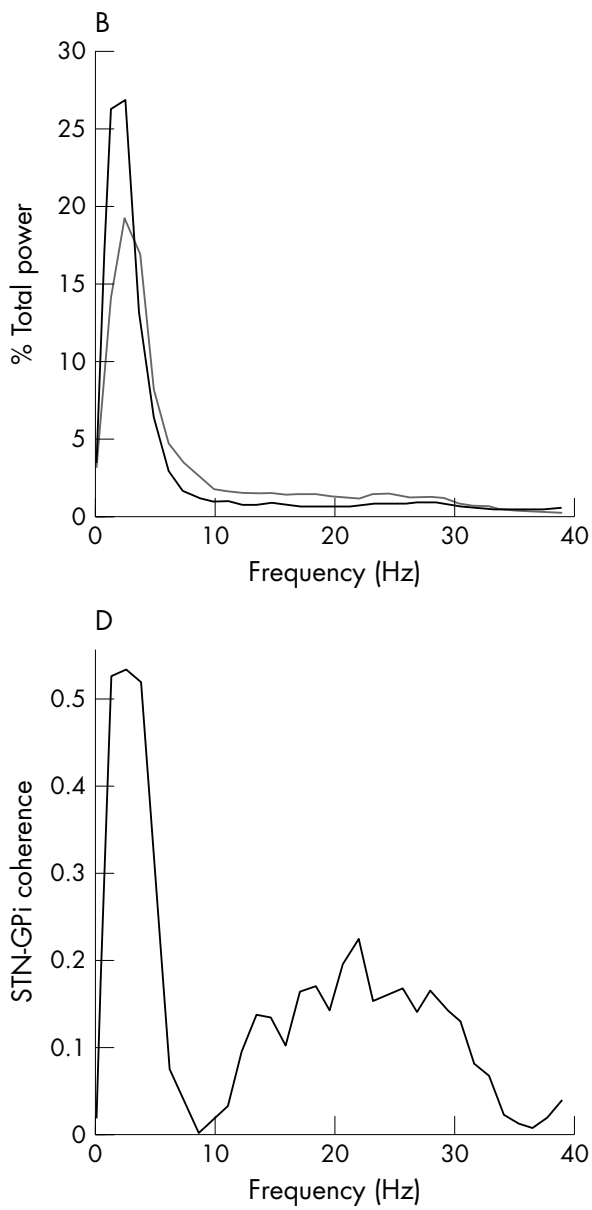

Figure 1 STN and GPi autospectra and STN-GPi synchronisation. (A) Autospectrum from the left nuclei ipsilateral to the dyskinetic side of the body. (B) Autospectrum from the right nuclei, contralateral to the dyskinetic side of the body. For A and $B$ the spectra are plotted as \% of the total power between 0 and $40 \mathrm{~Hz}$; grey line: STN; black line: GPi. Note that whilst the STN spectrum is symmetrical between the two sides, the right GPi spectrum has lower beta activity and higher low-frequency activity than the left spectrum, in line with previous findings in torsion dystonia. ${ }^{17}$ (C) STNGPi coherence in the left nuclei ipsilateral to the dyskinetic side of the body at rest (solid line) and during tonic contralateral voluntary contractions (dotted line). (D) STN-GPi coherence in the right nuclei contralateral to the dyskinetic side of the body. The same number of signal epochs was employed to estimate the coherence in the three conditions; therefore, the absolute values of coherence are comparable. The threshold for significance is 0.035 ( $p<0.001$; see Methods). Note that in the right nuclei contralateral to the dyskinetic side of the body the STN-GPi peak coherence decreased in the highbeta range $(20-30 \mathrm{~Hz})$ but remarkably increased at low frequencies $(<10 \mathrm{~Hz})$. (version 1.80). All further analyses were conducted off-line with Matlab software (version 6.5).

\section{Coherence analysis}

The synchronisation between the nuclei was analysed through coherence analysis. The coherence, which is a function of frequency with values between 0 and 1 that indicate how well two signals match at each frequency, was estimated from the LFPs of STN and GPi on $\sim 160 \mathrm{~s}$ long data segments at rest using Fourier analysis (Matlab routine "cohere"). Signals were divided into 195 sections of 2048 samples, with no overlap; each section was detrended and windowed by a Hanning window; the magnitude squared of the discrete Fourier transforms of the sections of the two signals was averaged to estimate the autospectra of the two nuclei $\left(\mathrm{P}_{\mathrm{STN}}\right.$ and $\left.\mathrm{P}_{\mathrm{GPi}}\right)$. The products of the discrete Fourier transforms of the sections of the two signals were averaged to form the cross-spectrum $\left(\mathrm{P}_{\mathrm{STN}-\mathrm{GPi}}\right)$. The coherence $\mathrm{C}_{\mathrm{STN}-\mathrm{GPi}}$ was given by $\mathrm{C}_{\mathrm{STN}-\mathrm{GPi}}=\left(\operatorname{abs}\left(\mathrm{P}_{\mathrm{STN}-\mathrm{GPi}}\right) .{ }^{\wedge} 2\right)$./ $\left(\mathrm{P}_{\mathrm{STN}}{ }^{*} \mathrm{P}_{\mathrm{GPi}}\right)$ and was considered significant $(\mathrm{p}<0.001)$ if it exceeded a threshold of 0.035 , as calculated by the formula $1-(1-\beta)^{1 /(L-1)}$, where $\beta$ is the confidence level $(\beta=0.999)$ and $\mathrm{L}$ is the number of sections $(\mathrm{L}=195) .{ }^{1322}$ The recordings and the analyses were conducted separately on both sides of the brain.

\section{RESULTS}

The pattern of STN and GPi autospectra was dominated by activity at frequencies $<10 \mathrm{~Hz}$ (fig $1 \mathrm{~A}$ and $\mathrm{B}$ ). Ipsilaterally to the dyskinetic side of the body at rest (fig lC, continuous line), the STN and GPi showed virtually no coherence at low frequencies but were well synchronised at high-beta frequencies $(20-30 \mathrm{~Hz}$; peak coherence $=\sim 10$ times the significance threshold at $\mathrm{p}<0.001$; see Methods). On the same side of the brain, contralateral voluntary muscle contractions reduced peak beta coherence and did not induce low frequency synchronisation (fig $\mathrm{IC}$, dotted line). Conversely, contralaterally to the dyskinetic side of the body (fig lD), the peak beta STN-GPi coherence was decreased at rest, but the bandwidth of the coherence actually increased, extending to low-beta frequencies $(10-20 \mathrm{~Hz})$. More strikingly, the nuclei contralateral to the dyskinetic side of the body were abnormally synchronised at low frequencies (peak coherence $=\sim 15$ times the significance threshold at $p=0.001)$. No peaks in the autospectra or in the coherence were observed at $\sim 70$ or $\sim 300 \mathrm{~Hz}$.

\section{DISCUSSION}

In our patient a transient lesion effect due to electrode introduction lowered the threshold for drug induced dyskinesias. In the nuclei contralateral to the dyskinetic side of the body (in comparison with the nuclei contralateral to the parkinsonian non-dyskinetic side of the body), there was a reduced STN-GPi synchronisation of LFP oscillations at highbeta frequencies $(20-30 \mathrm{~Hz})$ and increased STN-GPi synchronisation at low frequencies $(<10 \mathrm{~Hz})$. As far as we know, this is the first GPi-STN LFP study in parkinsonian dyskinesias. Despite the possible limitations of single-case observations, our case has several interesting aspects: there were electrodes in each side of the brain both in the STN and the GPi; dyskinesias were confined to the left side of the body, so that the two sides of the brain could be compared; and the patient 
was not anaesthetised and was drug free for several hours Moreover, the pattern of STN-GPi synchronisation contralateral to the non-dyskinetic side was consistent with the STN-GPi synchronisation described in parkinsonian patients. ${ }^{11}$

Beta LFP oscillations are pathologically high in Parkinson's disease both in the $\mathrm{GPi}^{17}$ and the $\mathrm{STN}^{11}{ }^{13} 15$ and the two nuclei are synchronised at these frequencies. ${ }^{11}$ Levodopa reduces beta oscillations within and between nuclei. ${ }^{11} 131517$ In patients with dystonia, GPi beta oscillations are reduced even more than in parkinsonian patients after levodopa. ${ }^{17}$ Here we add a further piece to this picture, showing that even without levodopa administration STN-GPi high-beta synchronisation is lower-but apparently wider-in parkinsonian dyskinesias compared to non-dyskinetic Parkinson's disease. This finding is in agreement with the antikinetic interpretation of beta oscillations. ${ }^{23}$ In addition, the different patterns of STN-GPi synchronisation we observed at highbeta and low-beta frequencies support the hypothesis that two different rhythms could operate in the beta band. ${ }^{12} 1415$

Low-frequency LFP oscillations in Parkinson's disease increase after dopaminergic medication both in the STN ${ }^{15}$ and the GPi. ${ }^{17}$ Dopaminergic medication does not induce lowfrequency STN-GPi synchronisation. ${ }^{11}$ In patients with dystonia, GPi low-frequency oscillations are increased more than in parkinsonian patients after levodopa. ${ }^{17}$ Here we show that, differently from treated or untreated Parkinson's disease, parkinsonian dyskinesias can be characterised by extremely strong STN-GPi low-frequency synchronisation. It should be noted that changes in coherence at frequencies $<10 \mathrm{~Hz}$ might potentially include movement related artefacts, ${ }^{1724}$ but the presence of synchronisation only in the dyskinetic nuclei makes this possibility unlikely. Hence, although several other possible factors might have contributed-at least partly-to coherence asymmetry, we believe that STN-GPi low-frequency synchronisation reflects a specific pathophysiological feature of parkinsonian dyskinesias.

According to the classical basal ganglia model, ${ }^{25}$ hyperkinetic symptoms arise from pathologically low firing rates in the STN and GPi, whereas hypokinetic symptoms arise from pathologically high firing rates in the STN and GPi. Thus, the coexistence of hypokinetic and hyperkinetic symptoms, as in our patient, leads the classical model to a paradox. Firing abnormalities within individual basal ganglia nuclei are not enough to account for the compound phenomenology of parkinsonian symptoms and more complex interactions between nuclei should be considered. As a possible solution of the paradox, our observations suggest that parkinsonian dyskinesias could arise not only from abnormal firing patterns within the GPi and the STN, ${ }^{3-6}$ but also from an altered synchronisation between these and, possibly, other basal ganglia nuclei.

\section{ACKNOWLEDGEMENTS}

The authors whish to thank S Garlaschi, G Gherardi, C Marcias, A Marsilio, M Pastori, and A Pellati for their technical assistance.

\section{Authors' affiliations}

G Foffani, G Ardolino, M Egidi, P Rampini, A Priori, Department of Neurological Sciences, Università di Milano, IRCCS Ospedale Maggiore di Milano, 20122 Milan, Italy

B Meda, G Baselli, Department of Biomedical Engineering, Politecnico di Milano, 20133 Milan, Italy

E Caputo, Department of Clinical Neurology, Ospedale San Paolo, 20142 Milan, Italy

This study was supported by the IRCCS Ospedale Maggiore di Milano, the Centro Dino Ferrari for Neurodegenerative Disorders, the Ministero della Sanità, and the Ministero dell'Università e della Ricerca Scientifica e Tecnologica. G Foffani was partially supported by the "Professor Guglielmo Scarlato Award" 2003 from the Società Italiana di Neurologia (S.I.N.). A Priori was partly supported by travel grant N00014-04-1-4004 from the US Department of the Navy, Office of Naval Research International Field Office.

Competing interests: none declared

Correspondence to: Professor Alberto Priori, Dipartimento di Scienze Neurologiche, Clinica Neurologica, Padiglione Ponti, Ospedale Maggiore Policlinico, Via F. Sforza 35, Milano, 20122 Italy; alberto.priori@unimi.it

Received 19 April 2004

In revised form 8 June 2004

Accepted 25 June 2004

\section{REFERENCES}

1 Marsden CD, Parkes JD. "On-off" effects in patients with Parkinson's disease on chronic levodopa therapy. Lancet 1976;1:292-6.

2 Obeso JA, Grandas F, Vaamonde J, et al. Motor complications associated with chronic levodopa therapy in Parkinson's disease. Neurology 1989;39: 11-9.

3 Obeso JA, Rodriguez-Oroz MC, Rodriguez M, et al. Pathophysiology of levodopa-induced dyskinesias in Parkinson's disease: problems with the current model. Ann Neurol 2000;47:S22-32.

4 Filion M. Physiologic basis of dyskinesia. Ann Neurol 2000;47:S35-40.

5 Vitek JL, Giroux M. Physiology of hypokinetic and hyperkinetic movement disorders: model for dyskinesia. Ann Neurol 2000;47:S131-40.

6 Bezard E, Brotchie JM, Gross CE. Pathophysiology of levodopa-induced dyskinesia: potential for new therapies. Nat Rev Neurosci 2001;2:577-88.

7 Papa SM, Desimone R, Fiorani M, et al. Internal globus pallidus discharge is nearly suppressed during levodopa-induced dyskinesias. Ann Neurol 1999:46:732-8.

8 Lozano AM, Lang AE, Levy R, et al. Neuronal recordings in Parkinson's disease patients with dyskinesias induced by apomorphine. Ann Neurol 2000;47:S141-6.

9 Boraud T, Bezard E, Bioulac B, et al. Dopamine agonist-induced dyskinesias are correlated to both firing pattern and frequency alterations of pallidal neurones in the MPTP-treated monkey. Brain 2001;124:546-57.

10 Levy $\mathbf{R}$, Dostrovsky JO, Lang AE, et al. Effects of apomorphine on subthalamic nucleus and globus pallidus internus neurons in patients with Parkinson's disease. J Neurophysiol 2001;86:249-60.

11 Brown P, Oliviero A, Mazzone P, et al. Dopamine dependency of oscillations between subthalamic nucleus and pallidum in Parkinson's disease. J Neurosci 2001;21:1033-8.

12 Foffani G, Priori A, Rohr M, et al. Event-related desynchronization (ERD) in the human subthalamus and internal globus pallidus. J Physiol 2002; 539:39P-40P

13 Levy R, Ashby P, Hutchison WD, et al. Dependence of subthalamic nucleus oscillations on movement and dopamine in Parkinson's disease. Brain 2002; 125: 1196-209.

14 Priori A, Foffani G, Pesenti A, et al. Movement-related modulation of neural activity in human basal ganglia and its L-DOPA dependency: recordings from deep brain stimulation electrodes in patients with Parkinson's disease. Neurol Sci 2002;23(suppl 2):S101-2.

15 Priori A, Foffani G, Pesenti A, et al. Rhythm-specific pharmacological modulation of subthalamic activity in Parkinson's disease. Exp Neurol 2004; 189:369-79.

16 Foffani G, Priori A, Egidi $\mathrm{M}$, et al. $300-\mathrm{Hz}$ subthalamic oscillations in Parkinson's disease. Brain 2003;126:2153-63.

17 Silberstein P, Kuhn AA, Kupsch A, et al. Patterning of globus pallidus local field potentials differs between Parkinson's disease and dystonia. Brain 2003; 126:2597-608

18 L. I.M.P.E. Guidelines for the treatment of Parkinson's disease. Neurol Sci 2003;24(suppl 3):S203-4

19 Egidi M, Rampini P, Locatelli M, et al. Visualisation of the subthalamic nucleus: a multiple sequential image fusion (MUSIF) technique for direct stereotaxic localisation and postoperative control. Neurol Sci 2002;23(suppl 2):S71-2.

20 Rampini PM, Locatelli M, Alimehmeti R, et al. Multiple sequential image-fusion and direct MRI localisation of the subthalamic nucleus for deep brain stimulation. J Neurosurg Sci 2003;47:33-9.

21 Priori A, Egidi M, Pesenti A, et al. Do intraoperative microrecordings improve subthalamic nucleus targeting in stereotactic neurosurgery for Parkinson's disease? J Neurosurg Sci 2003;47:56-60.

22 Rosenberg JR, Amjad AM, Breeze P, et al. The Fourier approach to the identification of functional coupling between neuronal spike trains. Prog Biophys Mol Biol 1989;53:1-31.

23 Brown P. Oscillatory nature of human basal ganglia activity: relationship to the pathophysiology of Parkinson's disease. Mov Disord 2003;18:357-63.

24 Manson AJ, Brown P, O'Sullivan JD, et al. An ambulatory dyskinesia monitor. J Neurol Neurosurg Psychiatry 2000;68:196-201.

25 Wichmann T, DeLong MR. Functional and pathophysiological models of the basal ganglia. Curr Opin Neurobiol 1996;6:751-8. 